\title{
COMENTARIOS SOBRE LA EFICACIA Y NEFROTOXI- CIDAD DE ANFOTERICINA B CONVENCIONAL E INTRALIPIDICA EN ADULTOS CON INFECCIÓN FÚNGICA SISTÉMICA Y COMPROMISO INMUNE
}

\section{(Comments on the effectiveness and nephrotoxicity of conventional and intralipidic Amphotericin B in immunocompromised adults with fungal systemic infections)}

\author{
Rodrigo Cruz Choappa. \\ Universidad de Valparaíso, Escuela de Medicina \\ Cátedra de Micología, Casilla 92V, Valparaíso, Chile
}

Palabras clave: Anfotericina B liposomal, eficacia, nefrotoxicidad Key words: Liposomal Amphotericin B, efficacy, nephrotoxicity

\section{RESUMEN}

En base a una revisión bibliográfica sobre el uso de anfotericina B liposomal y convencional, se determinó la diferencia en la eficacia y en la nefrotoxi-cidad entre ellas. Todos los estudios incluyeron pacientes inmunodeprimidos, neutropenicos y/o portadores de cáncer con diagnostico o alta sospecha de una infección fúngica sistémica. Para esta búsqueda se seleccionaron 9 estudios clínicos randomizados, doble ciego y un metaanálisis. Además se incluye un trabajo chileno en cohorte.

Ocho estudios muestran menor nefrotoxicidad en los pacientes tratados con Anfotericina B liposomal y 7 de los 10 estudios randomizados no muestran diferencias en la eficacia de estas drogas.

Las infecciones fúngicas sistémicas se han convertido en un problema serio y cada vez más frecuente entre pacientes inmunodeprimidos, transplantados y hospitalizados con factores de riesgo $(1,2)$.

Una infección fúngica sistémica en pacientes de este tipo es grave, por lo que se requiere un tratamiento oportuno y eficaz para combatirlas y así evitar un final catastrófico.

Un antifungico antiguo pero ampliamente utilizado en la actualidad es la Anfotericina B, antifúngico polié-nico que se aisló por primera vez en el año 1956 a partir de Streptomices nodosus. Convencionalmente se

\section{ABSTRACT}

Based on the revision of literature about the use of liposomal and conventional Amphotericin B, the difference in effectiveness and nephrotoxicity between them was determined. All studies included immuno-depressed, neutropenic patients and/or cancer carriers being diagnosed or highly suspected of a systemic fungal infection. In order to do this, nine randomised clinic studies, double blind and a methaanalysis were selected. Besides a chilean report on cohorte is inclu-ded. Eight studies revealed lower nephrotoxicity in patients treated with liposomal Amphotericin B whereas 7 out of 10 randomised studies showed no differences in the effectiveness of these drugs.

\section{INTRODUCCION}

ha administrado por vía endovenosa en suero glucosado al 5\% con buena respuesta clínica (3), sin embargo, su administración se puede asociar con efectos adversos inmediatos, tales como fiebre, calofríos, nauseas, vómitos, cefalea, shock anafiláctico, arritmias, falla hepática etc. y reacciones adversas que se presentan después de administraciones repetidas de la droga (hipokalemia, hipomagnesemia, toxicidad medular), sin embargo, la toxicidad más temida y en la cual se han centrado la mayoría de los estudios sigue siendo la nefrotoxicídad, la cual puede ser transitoria o irreversible (4). E n la actualidad existen las formulaciones lipídicas de la 
Anfotericina $\mathrm{B}$, las cuales disminuirían estas reacciones adversas y en especial la nefrotoxicidad, sin embargo, son de mayor costo económico y difíciles de conseguir en nuestro país. Dentro de estas formulaciones destaca la Anfotericina B liposomal (AMBL-Ambiso-me ${ }^{\circledR}$ ) con un $10 \%$ de Anfotericina en relación a los lípi-dos (5).

Existe en la actualidad una gran demanda para determinar el costo-eficacia de estas nuevas formu-laciones y la toxicidad de cada una de ellas en relación a la Anfotericina B tradicional. Este es un problema que ha ido creciendo y estudiándose fuertemente en los últimos 10 años, debido a su impacto clínico y económico para el paciente y las instituciones que imparten salud.

Por tal motivo nos planteamos la pregunta: ¿Es la Anfotericina B liposomal más efectiva y menos nefrotoxica que la Anfotericina convencional en infecciones fúngicas sistémicas en pacientes inmuno-deprimidos adultos?.

\section{MATERIALES Y METODOS}

Los estudios bibliográficos seleccionados debían cumplir con los sigientes requisitos para ser incluídos en este análisis.

Sujetos: pacientes adultos inmunodeprimidos, neutropenicos y/o portadores de cáncer con diagnóstico (cultivo en sangre de Candida u hongo filamentoso) o alta sospecha de infección fúngica sistémica. Se consideró de alta sospecha cuando un paciente presentó fiebre persistente a pesar de tratamiento antibiótico de amplio espectro (Vancomicina, Amikacina).

Se comparó la eficacia y nefrotoxicidad entre pacientes tratados con Anfotericina B convencional (en glucosa al 5\%) y la Anfotericina B liposomal (intralipidica al $20 \%$ ) en dosis de $0.75-2 \mathrm{mg} / \mathrm{kg}$ día. La infusión del medicamento fue en 1-2 hrs.

Definición de eficacia: ausencia de síntomas y/o evidencia de infección fúngica sistémica, eliminación de la fiebre por tres días consecutivos.

Definición de nefrotoxicidad: decrecimiento de un 50\% o más del clearence de creatinina o un incremento de $0,5 \mathrm{mg} / \mathrm{dl}$ de la creatinininemia desde el nivel basal. Se realizaron mediciones diarias.

Tipo de estudios: se seleccionaron solo ensayos clínicos randomizádos doble ciego y metaanalisis de ensayos clínicos doble ciegos.

Estrategia de búsqueda: búsqueda de publicaciones en PubMed, Biblioteca Cocrhane, Review of Medical and Veterinary Mycology, Revista Médica de Chile, literatura gris.

Resultado de la búsqueda: Se eligieron sólo los estudios clínicos que cumplieron con nuestros criterios y que compararan nefrotoxicidad y eficacia de la Anfotericina B lipo- somal versus la convencional. Se encontraron 10 estudios extranjeros de los cuales $\mathbf{1}$ corresponde a un metaanalisis. Además se incluye una experiencia nacional (cohorte).

\section{RESULTADOS Y ANALISIS CRITICO de los estudios seleccionados (resúmenes):}

Se seleccionaren 10 estudios que cumplían con las características para poder responder la pregunta formulada:

Estudio 1(6): año 1994-1995, TTO: dosis de 1mg/kg, 80\% de los pacientes con tratamiento Empírico, los grupos fueron de 33(grupo 1) y 28 (grupo 2) pacientes. Edad promedio: 19 (2-62).

\begin{tabular}{|l|c|c|c|}
\cline { 2 - 3 } \multicolumn{1}{c|}{} & Liposom al & Con rencional & \multicolumn{1}{c|}{} \\
\cline { 2 - 3 } \multicolumn{1}{c|}{ Mefrobo 1} & Grupo 2 & $\mathrm{p}$ \\
\hline Eficidad & Sin diferercias & 0.44 \\
\hline
\end{tabular}

Tanto en la nefrotoxicidad como en la eficacia el estudio revela que no existen diferencias entre ambos tratamientos, sin embargo, en la evaluación de la nefrotoxicidad este estudio cuenta con un P de 0.44 , lo que no lo valida estadísticamente. Además cuenta con la limitación de haber incluido niños, lo cual hace difícil su comparación con el resto de los estudios.

Estudio 2(7): año1995. 20 pacientes adultos por grupo (1 y 2). $75 \%$ de los tratamientos fue empírico. Abstract.

\begin{tabular}{|l|c|c|c|}
\cline { 2 - 4 } \multicolumn{1}{c|}{} & Liposomal & Con rencional & \multicolumn{1}{c|}{} \\
\cline { 2 - 4 } \multicolumn{1}{c|}{} & Grupo 1 & Grupo 2 & P \\
\hline Nefroboxicidad & Merur & Mayor & 0.047 \\
\hline Eficacia & \multicolumn{2}{|c|}{ Sin diferercias } & $\cdots$ \\
\hline
\end{tabular}

El estudio muestra que no existe diferencia en cuanto a la eficacia de los dos medicamentos, sin embargo los pacientes tratados con anfotericina B liposomal presentaron menor nefrotoxicidad, diferencia apoyada con un p de 0.047 .

No se muestra el $\mathrm{P}$ al evaluar la eficacia y se recalca lo difícil de esta medición debido a que en su mayoría los tratamientos fueron empíricos.

Estudio 3(8): año 2000. 134 pacientes adultos divididos en 2 grupos. 59 pacientes con infección fúngica documentada. 


\begin{tabular}{|c|c|c|c|}
\hline & Liponond & Domr ncions I & \\
\hline & Grups 1 & Grupol & $\mathbf{F}$ \\
\hline He Enotor ix d d & hitsux & $\operatorname{lithyor}$ & 0,000 \\
\hline Ebiodid & \multicolumn{2}{|c|}{ 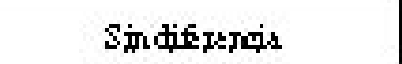 } & 00 \\
\hline
\end{tabular}

Este estudio demuestra que no existe diferencia en cuanto a eficacia entre las Anfotericinas, sin embargo, si la hay en lo referente a la nefrotoxicidad, la cual es menor en la liposomal. Es un estudio estadísticamente significativo.

Estudio 4(9): año 1999. El grupo 1 consto con 341 pacientes v el ? con 342

\begin{tabular}{|c|c|c|c|}
\hline \multirow[t]{3}{*}{. } & \multirow{2}{*}{ Liponal } & \multirow{2}{*}{ Com nciond I } & \\
\hline & & & \\
\hline & Grupo 1 & Grupot & F \\
\hline He Entorinids d] & MLLTUT & hisyor & \\
\hline E.6ios cid & \multicolumn{2}{|c|}{ 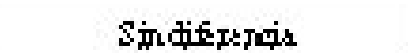 } & \\
\hline
\end{tabular}

Estudio con un $\mathrm{n}$ importante, con validez estadística que demuestra que la Anfotericina liposomal presentando la misma eficacia que la tradicional tiene a la vez menor nefrotoxicidad.

Estudio 5(10): año 2002. Metaanalisis de 3 ensayos clínicos ( 1149 pacientes adultos) donde se comparó las dos drogas.

\begin{tabular}{|c|c|c|c|}
\hline & Lipsomd & Comr ncions I & \\
\hline & Grupo 1 & Grupol & F \\
\hline 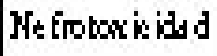 & hlowor & lityor & $0 \pi$ \\
\hline Efousid & hloys & HLTUT & 00 \\
\hline
\end{tabular}

En este metaanalisis se demuestra con buena homo-geneidad y validez estadística que la Anfotericina B liposomal, es más efectiva y menos nefrotoxica que la formulación convencional. Intervalo de confianza de $95 \%$.

Estudio 6(11): año 1994. Dos grupos de 21 pacientes adultos. Ambos grupos recibieron antibióticos de amplio espectro.

\begin{tabular}{|c|c|c|c|}
\hline & Liposomal & Comencions 1 & \\
\hline & Grupo 1 & Erupo 2 & F \\
\hline He totaris id d & hLțWT & 16yor & $0[01$ \\
\hline E.jos cid & \multicolumn{2}{|c|}{ 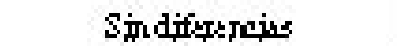 } & $=0,005$ \\
\hline
\end{tabular}

Trabajo francés que no muestra diferencias en cuanto a eficacia de las dos drogas, pero si en la nefrotoxicidad, siendo menor en el caso de la liposomal. Estadísticamente valido.

Estudio 7(12): año1992. 22 pacientes divididos en dos grupos, a los cuales se les administró las drogas del estudio.

\begin{tabular}{|c|c|c|c|}
\hline & Lipoomd & Comencions I & \\
\hline & Grupo 1 & Grupo: & F \\
\hline Petobrind d & 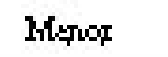 & hityor & 0,001 \\
\hline E. & \multicolumn{2}{|c|}{ 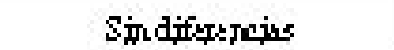 } & 0,0 \\
\hline
\end{tabular}

Los resultados del trabajo coinciden en su gran mayoría con el resto de los estudios, sin embargo no describe el P ni otros test de significación estadística. Además presenta un $\mathrm{n}$ pequeño. Como trabajo único no puede ser considerado, pero dentro de una búsqueda sistemática puede ser valioso su aporte.

Estudio 8(13): año 2002. Se estudiaron 2 grupos de 17 pacientes adultos.

\begin{tabular}{|c|c|c|c|}
\hline & Liponomsl & Comencion I & \\
\hline & Erupo 1 & Grupo: & F \\
\hline Ye Entorin its d & MLTUOT & lithyor & 0005 \\
\hline E6osid & hitopor & likTOS & 005 \\
\hline
\end{tabular}

Estudio cuyos resultados muestran con validez estadística que la Anfotericina B liposomal presenta menor nefrotoxicidad que la Anfotericina convencional.

Estudio 9(14): año 2002. 22 pacientes adultos (grupo 1) fueron tratados con Anfotericina convencional y 51 (grupo 2) con Anfotericina B liposomal . Promedio de edad: 33(16-68). 
7. Pascual, B.; Ayestaran, A.; Montoro, J.B.; Olivera, J.; Estibalez, A.; Julia, A.; Lopez, A. (1995). Administration of lipid-emulsion versus conventional amphotericin B in pacients with neutropenia. Ann Pharmacother. 29:1197-201

8. Blau, I.W.\& Fauser, A.A.(2000).Comparative studies betwen conventional and liposamal amphotericin B in neutropenic patients with fever unknown origin and patients with systemic mycosis. Mycoses 43: $325-332$.

9.Walsh ,T.J.; Finberg, R.; \& Arndt, C. (1999).Liposomal amphotericin $\mathrm{B}$ for empirical therapy in patients with persistent fever and neutropenia. New England Journal of Medicine 340:764-771

10. Johansen, H.K. \& Gotzsche, P.C. (2002). Amphotericin B lipid soluble formulation versus amphotericin $\mathrm{B}$ in cancer patients with neutropenia (Cochrane review). Cochrane Database Syst. Rev.Cd000329.

11. Caillot, D.; Reny, G.; Solary, E. ; Casasnovas, O.; Chavanet, P.; Bonnotte, B.; Perello, L. et al., (1994).A controlled of the tolerante of amphotericin B infused in dextroxa or in intralipid in patients with haematological malignancies. Journal of Antimicrobial Chemotherapy 33: 603- 613

12. Chavanet, P.Y.; Garry, L.; Charlier, N.; Caillot, D.; Kisterman, J.P.; D'Athis, M.; Portier, H. (1992). Trial of glucose versus fat emulsion in preparation of amphotericin for use in HIV patients with candidiasis. Br.J.Med. $305: 921-925$

13. Bodhe, P.V.; Kotvani, R.N.; Kirodian, B.G.; Kshirsagar, N.A .; Pandya, S.K. (2002). Randomized, comparative phase III safety and efficacy study with conventional amphotericin B and liposomal amphotericin B in patients with sytsemic fungal infection. Assoc. physicians India 50:662-670
14. Philip, C.J.;Weat, L.J.; Gretchen, A. C.; Goldman, M. ; Lancaster, D.; Bamberger, D.; Powderly, W. et al.(2002).Safety and efficacy of liposomal amphotericin B compared with conventional ampotericin B for induction therapy of hisptoplasmosis in patients AIDS. Ann. Inter. Med. 137:105-109

15. Schoffki, P.; Freund, M.; Wunder, R.; Kohne, CH. H. (1998). Safety and toxicity of amphotericin B in glucose $5 \%$ or intralipid $20 \%$ in neutropenic patients with pneumonia of unknown origin: randomized study. B.M.J. $\quad 317: 379-384$

16. Hernandez, G.; Altermatt, F.; Bernucci, F. (2000).Uso de la Anfotericina B en mezcla lipídica: ¿previene la toxicidad de la droga en pacientes críticos?. Revista Médica de Chile. 128:1101-1107

17. Thompson, L. (1997). Anfotericina B: nuevas formas farmacéuticas. Rev. Chil. Infect. 14:258-262 


\begin{tabular}{|c|c|c|c|}
\hline & Lipond & Comencions 1 & \\
\hline & Grupol 1 & Grupo 2 & F \\
\hline Je trotos is ids d & Mt:Lugr & hthyor & 000 \\
\hline E. biosid & MLopor & MtLTET & 0.014 \\
\hline
\end{tabular}

Excelente trabajo, con sígnificancia estadística (P menor a 0.05 ), intervalos de confianza óptimos, un $\mathrm{n}$ adecuado. Demuestra la mayor eficacia y la menor toxicidad de la Anfotericina B liposomal en los pacientes portador de VIH- SIDA con infección por Histoplasmosis.

Estudio 10(15): año 1998. 51 pacientes neutropenicos divididos en 2 grupos.

\begin{tabular}{|c|c|c|c|}
\hline & Lipoomsl & Comencions I & \\
\hline & Grupo 1 & Grupo 2 & F \\
\hline Pe Emborinids & \multicolumn{2}{|c|}{ 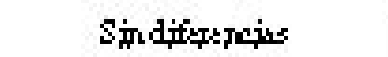 } & 000 \\
\hline Ebios cid & \multicolumn{2}{|c|}{ Wo tis sulphats } & 0.014 \\
\hline
\end{tabular}

Estudio con validez estadística, compara dos grupos igualados en la edad, sexo, comorbilidad, función renal, tratamiento antibióticos y fármacos nefrotoxicos.

Muestra que no existen diferencias en cuanto a nefrotoxicidad entre los dos fármacos. En este estudio no se comparó la eficacia entre los fármacos.

Experiencia chilena (16): estudio realizado entre los años 1996-1997 en dos grupos; uno de 17 pacientes (tratados con Anfotericina convencional) y otro de 28 pacientes (tratados con Anfotericina B liposomal) .Todos los pacientes fueron sometidos a un protocolo estandarizado y a la escala de gravedad APACHE II. En 20 de los casos la terapia antifúngica se inicio empíri-camente.

Este estudio no muestra diferencias en prevención de la nefrotoxicidad ni en la eficacia entre ambas formulaciones aplicadas en grupos comparables, sin embargo, al realizar un análisis crítico de este, encontramos que no hubo aleatorización, no hubo doble ciego, no se describe exclusión de pacientes ni seguimiento, además de no presentar la validez estadística para las medidas de nefrotoxicidad y eficacia. Por tal motivo es difícil considerar la magnitud del efecto y la aplicabilidad en los pacientes.
De los $\mathbf{1 0}$ trabajos analizados en esta revisión, $\mathbf{8}$ muestran menor nefrotoxicidad en los pacientes que fueron tratados con Anfotericina B liposomal versus aquellos pacientes tratados con Anfotericina conven-cional. Solo 2 estudios no muestran diferencias en cuanto a nefrotoxicidad, pero el estudio 1, en relación a esta medida tiene un $\mathrm{P}$ de 0.44 , lo cual lo invalida estadísticamente.

Con respecto a la eficacia de los 2 fármacos estudiados, 7 muestran que no existe mayor diferencia entre la Anfotericina liposomal y al tradicional, 2 estudios muestran mayor eficacia de la Anfoterricina liposomal y 1 no evaluó dicha medida. Debe considerarse que la mayor parte de los tratamientos fueron de tipo empírico, razón por la cual se hace difícil poder evaluar la eficacia considerando si realmente se trató de una infección fúngica. Los grupos dentro de cada estudio eran comparables en cuanto a edad, comorbilidad, tratamientos adjuntos, fármacos nefrotoxicos, etc. además recibieron la misma dosis de antifúngico dentro del estudio al cual pertenecían.

Recomendaciones basadas en la evidencia encontrada: Con las pruebas acumuladas en este trabajo creemos que, se puede sugerir como tratamiento de elección la Anfotericina B liposomal (si se encuentra disponible y si se tienen los recursos económicos), para los pacientes inmunodeprimidos que presenten una infección fúngica sistémica, ya sea comprobada o de alta sospecha, con el fin de evitar la tan temida nefrotoxicidad y al menos con la misma eficacia que la Anfotericina B convencional. Una alternativa que Thomson (1997)(17), recomienda en pacientes en los cuales la anfortericina convencional ha fracasado tras 7 o 10 días de empleo o en pacientes con falla renal.

\section{REFERENCIAS}

1. Grau, S.; Torres, J.\& Monterde, J. (1996). Situación actual de las infecciones por hongos. Farm Hosp. 1996; 20(6): 390-392.

2. Porton , Jose. (2003). Aspergillosis Invasora. Primera Edición. 2003. España.Pfizer.

3. Lyman, C.A. \& Walsh, T.J. (1992). Systemically administered antifungal agents. A review of their fharmacology and therapeutic applications. Drugs 44:9-35

4. Luke, R.G. \& Boyle, J.A. (1998).Renal effects of Amphotericin B. Am .J .Kidney Dis. 31:780-5

5. Ranchere ,J.Y.; Latour, J.F.; Fuhrmann, C.; Lagallarde, C.;Loreuil, F. (1996).Amphotericin B intralipid formulacion: Stability and particle size. J.Antimicrob. Chemother. 37: 1165-1169

6. Nucci, M.; Loureiro, M.; Silveira, F.; Casali, A.R.; Bouzas, L.F.; Velazco, E.; Spector, N. et al., (1999).Comparaison of toxicity of anfotericin B in 5\% dextrose with that of amphotericin B in fat emulsion in a randomized trial with cancer patients. Antimicrobial agents and chemotheraphy. 43:1445-1448 